\title{
Escherichia coli Pyomyositis Presenting as Right Hip Pain - a Case Report and Review of Literature
}

\author{
Mark Sigler MD, John Midturi DO, MPH, Timothy Byrd MD
}

\begin{abstract}
Pyomyositis typically occurs after hematogenous bacterial dissemination, and methicillin-resistant Staphylococcus aureus (MRSA) and Group A Streptococci are the most common organisms isolated. Potential contributing factors to pyomyositis include underlying malignancy, trauma, or concurrent infection at other sites. In this case report, we present a 58-year-old woman with two months of fevers and two weeks of right hip pain who was ultimately diagnosed with pyomyositis secondary to Escherichia coli.
\end{abstract}

Key words: sepsis, pyomyositis, Escherichia coli

\section{INTRODUCTION}

Hip pain is a common symptom (up to $15 \%$ of adults greater than 60 -years-old report hip pain within the past 6 weeks), and the differential diagnosis is exceptionally broad. ${ }^{1}$ Trochanteric bursitis, gluteus medius bursitis, hip osteoarthritis, meralgia paresthetica, osteonecrosis, occult hip fracture, aortoiliac vascular occlusive disease, and referred pain are relatively common causes of hip pain. ${ }^{2,3}$ As described below, pyomyositis is a much less common etiology of hip pain but should be considered in the differential diagnosis.

Corresponding author: Mark Sigler MD

Contact Information: mark.sigler@ttuhsc.edu DOI: 10.12746/swrccc2014.0205.062

\section{Case Presenttion}

A 58-year-old Caucasian woman presented with a two-month history of subjective fevers and a two-week history of progressive right hip pain, weight loss, and night sweats. The patient's only past medical history was COPD (on no chronic medications). On presentation, she had a temperature of $103^{\circ} \mathrm{F}$, pulse 114 beats per minute, respiratory rate 20 breaths per minute, and blood pressure 114/62 mmHg. Physical examination revealed diffuse tenderness to palpation on the proximal anteromedial aspect of the right thigh with associated swelling and erythema. Laboratory studies showed a white blood cell count of $19 \mathrm{k} / \mu \mathrm{L}$, ESR 109 mm/hour, and CRP 326 mg/dl. Initial radiographs demonstrated a foreign body on the medial aspect of her right lower extremity (Figure). An MRI of her right lower extremity revealed a multi-loculated fluid collection around a metallic fragment in the hamstring compartment. Pyomyositis was suspected, and incision and drainage was performed with foreign body removal. Cultures obtained during surgery grew Escherichia coli. The patient was treated with Ertapenem after return of susceptibilities. After discovery of 
the foreign body, the patient recalled that 30 years ago she had been mowing her yard and felt a "bite" at the location of the foreign body. However, she thought this was a trivial injury at the time, and no further investigation was done.

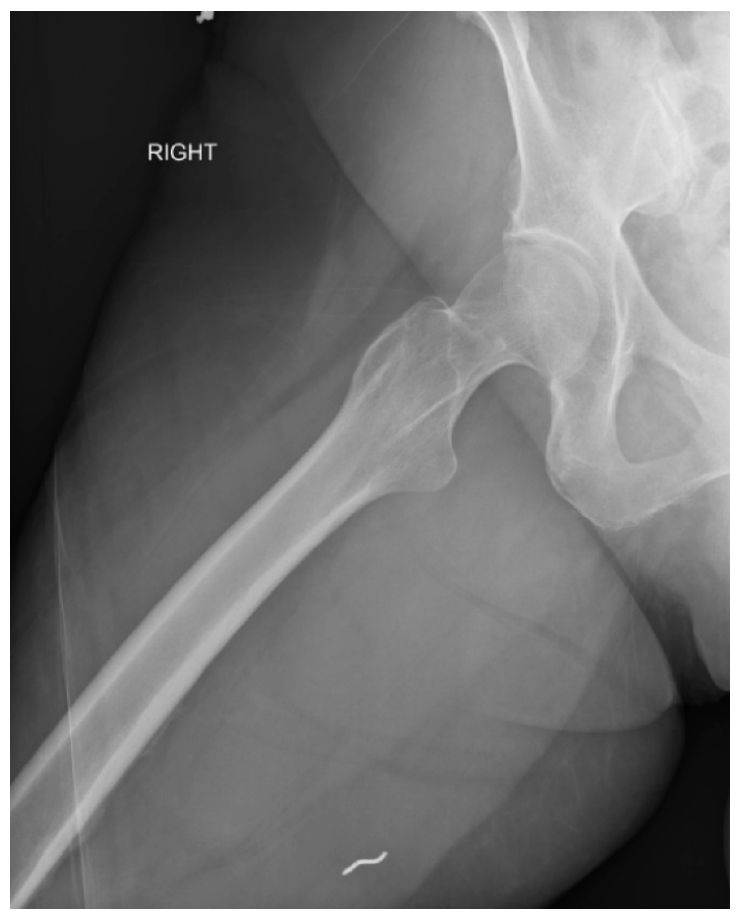

Figure: Plain radiograph of the right hip demonstrating a foreign body in the soft tissues on the medial aspect of the thigh.

\section{Discussion}

Pyomyositis is an intramuscular abscess in large skeletal muscles; it develops in the clinical context of transient bacteremia with the presence of concurrent muscle injury. ${ }^{4}$ Pyomyositis has three distinct phases: 1) diffuse muscle infection, 2) abscess formation, and 3 ) sepsis. These phases typically occur sequentially, and the diagnosis is often difficult during the initial phases. Based on clinical and laboratory data, our patient had progressed to the third phase (sepsis) at the time of her presentation.
Pyomyositis usually develops as a result of hematogenous spread; the most common causative organisms are MRSA and Group A Streptococci. Previously, it was thought that it was a disease found in tropical climate zones. However, more recent studies have demonstrated that pyomyositis is not limited to the tropics, and it may develop in immunocompromised patients in temperate climates. ${ }^{5}$ Since the first reported case of pyomyositis in 1971, there have been over 300 reported cases of pyomyositis in the United States. ${ }^{6}$ There were 246 cases reported between 1981 and 1994 in HIV-negative patients; these included 24 cases with Gram-negative bacteria.

Twenty cases of Escherichia coli pyomyositis have been reported, with nearly all of them occurring in immunocompromised patients. ${ }^{7}$ In 2010, Clinical Infectious Diseases published an article by Vigil et al. that reported an emerging trend of Escherichia coli pyomyositis in patients with hematologic malignancies. Six cases at MD Anderson Cancer Center were identified between 2003 and 2007; five of the six patients had calf involvement. All had an underlying hematologic malignancy with either severe neutropenia or were receiving high-dose corticosteroids. Fifty percent of these patients required admission to the intensive care unit for hypotension, and there was a $33 \%$ mortality rate. ${ }^{8}$ Similar to our patient presented in this report, the clinical course of E. coli pyomyositis typically begins with a subtle clinical presentation including pain and tenderness of the involved area followed by development of low-grade fever and leukocytosis. ${ }^{8}$ An MRI is considered the most reliable diagnostic test, and the MRI performed of our patient demonstrated a multi-loculated fluid collection in the hamstring compartment. ${ }^{9}$ Treatment includes complete drainage of any abscess cavities combined with appropriate antibiotic therapy, and most patients have a complete recovery without long-term complications or sequelae.

In summary, pyomyositis remains a rare infection, and Escherichia coli is an unusual pathogen causing this infection. We report a patient with Escherichia coli pyomyositis who presented in the third 
phase (sepsis); this patient responded well to the recommended treatment of drainage of the abscess with appropriate antibiotic therapy. This case highlights the importance of maintaining a high clinical suspicion for pyomyositis in patients presenting with fever and joint/muscle pain. These patients should be evaluated with an MRI and respond well to surgery and antibiotics.

\section{KEYPOINTS}

1. MRSA and Group A Streptococci are the most common organisms isolated in patients with pyomyositis.

2. Contributing factors to development of pyomyositis include underlying malignancy, trauma, and concurrent infection at other sites.

3. MRI is the most reliable diagnostic test for pyomyositis.

4. Treatment of pyomyositis includes complete drainage combined with antibiotics.

Author Affiliation: Mark Sigler is a fellow in pulmonary and critical care medicine in the Department of Internal Medicine at TTUHSC in Lubbock. John Midturi and Timothy Byrd work in the Department of Internal Medicine at Texas A \& M/Scott and White Memorial Hospital.

Received: $10 / 11 / 2013$

Accepted: 01/03/2014

Reviewers: Edward Pesanti MD, Michael Phy DO

Published electronically: 01/15/2014

Conflict of Interest Disclosures: None

\section{REFERENCES}

1. Christmas C, Crespo CJ, Franckowiak SC, et al. How common is hip pain among older adults? Results from the Third National Health and Nutrition Examination Survey. J Fam Pract 2002; 51:345-348.

2. Anderson BC. Office Orthopedics for Primary Care: Diagnosis and Treatment. 2nd, WB Saunders, Philadelphia 1999.

3. Anderson BC. Evaluation of the adult with hip pain. http:// www.uptodate.com/contents/evaluation-of-the-adult-with-hippain. Accessed 12/11/2013.

4. Crum NF. Bacterial pyomyositis in the United States. Am J Med 2004; 117:420-428.

5. Hsueh PR, Hsiue TR, Hsieh WC. Pyomyositis in intravenous drug abusers: report of a unique case and review of the literature. Clin Infect Dis 1996; 22:858-860.

6. "Tropical" pyomyositis: an unusual infection due to Staphylococcus aureus. N Engl J Med 1971; 284:196-198.

7. Masferrer-Pino A, et al. Pyomyositis of the inner thigh muscles due to Escherichia coli in a young patient with severe aplastic anemia. Rheumatol Clin. 2013; http://dx.doi.org/10.1016/j.reuma. 2013.01.003

8. Vigil, Karen J; Johnson, James R; Johnston, Brian D et al. Escherichia coli pyomyositis: an emerging infectious disease among patients with hematologic malignancies. Clin Infect Dis 2010; 50:374-380.

9. Bickels J, Ben-Sira L, Kessler A, Wientroub S. Primary pyomyositis. J Bone Joint Surg Am 2002; 84-A: 2277-86. 